\title{
Perspective
}

\section{SPATA2: more than a missing link}

\author{
Lisa Schlicher ${ }^{1,2,3}$, Prisca Brauns-Schubert ${ }^{1,2,3}$, Florian Schubert $^{1,2,3}$ and Ulrich Maurer ${ }^{*, 1,2,3}$
}

The assembly of the TNFR1 signalling complex (TNF-RSC) depends on K63- and M1-linked ubiquitylation, promoting the recruitment of complex constituents and the stability of the complex. Ubiquitylation is a dynamic process, controlled by E3 ubiquitin ligases as well as deubiquitinases, such as CYLD and OTULIN. A novel molecule, SPATA2, which is crucial for recruiting and activating the deubiquitinase CYLD within the TNF-RSC, has now been identified by four different studies. Loss of SPATA2 was shown to result in increased TNF-, but also NOD2-mediated proinflammatory signalling. Importantly, SPATA2 is instrumental for TNF-induced cell death, and a closer look at these findings suggests that SPATA2 possibly has functions beyond promoting the activity of CYLD.

Cell Death and Differentiation (2017) 24, 1142-1147; doi:10.1038/cdd.2017.26; published online 10 March 2017

Inflammation, triggered by pathogens or by tissue damage, is mediated by the local action of proinflammatory cytokines such as TNF and IL-1 $\beta$ expressed by cells of the innate immune response. These molecules trigger the typical effects like swelling, redness, heat, and pain, which are the consequences of locally increased blood flow and vessel permeability. The initial recognition of pathogen associated molecular patterns (PAMPs) and danger associated molecular patterns (DAMPs) by pattern recognition receptors (PRRs) such as Toll-like receptors (TLRs), NOD-like receptors (NLRs) and RIG1-like receptors (RLRs) induces the expression of proinflammatory cytokines, and activates antigen-presenting cells (APCs), thereby enabling subsequent adaptive immune responses. ${ }^{1}$

Proinflammatory signalling pathways, induced by cytokine receptors and PRRs, have been subject to extensive research within recent years, and it was recognised that ubiquitylation plays a key role for inflammatory signalling. Ubiquitin is a small (76aa) protein, which is linked by the coordinated action of an E1 ubiquitin activating enzyme, an E2-conjugating enzyme and an E3 ubiquitin ligase to substrate proteins and/or ubiquitin itself. Thereby, the C-terminal glycine of ubiquitin is covalently linked to the epsilon-amino group of a lysine in the target protein, generating an iso-peptide bond.

Target proteins can be mono-or poly-ubiquitylated, mediating diverse effects in cells such as protein degradation, receptor assembly and signalling, or the regulation of transcription. There are a number of lysines in the aminoacid sequence of ubiquitin, which can be target for the attachment of the C-terminus of another ubiquitin, such as $\mathrm{K} 6$, $\mathrm{K} 11, \mathrm{~K} 27, \mathrm{~K} 29, \mathrm{~K} 33, \mathrm{~K} 48$, and $\mathrm{K} 63$. In addition, the N-terminal methionine (M1) can be linked to the C-terminal glycine of another ubiquitin by a peptide bond, resulting in a linear ubiquitin linkage. In addition, polyubiquitin chains with mixed linkages also exist. ${ }^{2,3}$ Ubiquitin chains are generated by more than 500 E3 ligases, with different protein targets and linkage specificities. While K48-linked ubiquitylation is a signal for protein degradation by the proteasome, K63- and M1-linked polyubiquitin chains were shown to be instrumental in providing the scaffold, which promotes the assembly and stability of immune receptor complexes.

TNF signalling represents a prototype proinflammatory pathway where these biochemical principles are applied. Thus, for TNF signalling, degradative, K48-linked ubiquitylation, and the non-degadative K63- and M1-linked ubiquitylations play a central role. The current model of this pathway holds that, upon ligand binding, TNFR1 trimerisation induces the formation of a protein complex (also termed complex I). Thereby, TRADD and RIPK1 are recruited through death domain interactions, followed by the recruitment of the factors TRAF2, and clAP1/2, which mediate K63-linked ubiquitylation of RIPK1. K63-linked ubiquitin chains are required to recruit the adapters TAB $1 / 2$ along with the kinase TAK $1 .{ }^{4}$ In addition, K63-linked ubiquitylation results in the recruitment of the LUBAC complex, which is consisting of the proteins SHARPIN, HOIL-1, and the E3 ligase HOIP. LUBAC meditates M1-linked (linear) ubiquitylation of various complex components, such as NEMO and RIPK1, resulting in further stabilisation of the receptor complex. Linear ubiquitylation is crucial for the recruitment and activation of the IKK complex through the interaction of the adaptor NEMO with M1- linked ubiquitin chains. ${ }^{5}$ The activity of the kinases IKKa and IKK $\beta$ promotes the K48-linked ubiquitylation and subsequent degradation of $\mathrm{I}_{\kappa} \mathrm{B} a$, allowing $\mathrm{NF}-\kappa \mathrm{B}$ transcription factor activity, while TAK1 activates IKKs and MAPK kinases, together resulting in the transcriptional induction of proinflammatory target genes.

${ }_{1}^{1}$ Institute of Molecular Medicine and Cell Research, Albert-Ludwigs-University Freiburg, Stefan Meier Strasse 17, Freiburg 79104, Germany; ${ }^{2}$ Spemann Graduate School of Biology and Medicine (SGBM), Albert-Ludwigs-University of Freiburg, Albertstrasse 19a, Freiburg 79104, Germany and ${ }^{3}$ BIOSS, Centre for Biological Signaling Studies, Hebelstrasse 2, Freiburg 79104, Germany

${ }^{*}$ Corresponding author: U Maurer, Institute of Molecular Medicine and Cell Research, Albert-Ludwigs-University Freiburg, Stefan Meier Strasse 17, Freiburg 79104, Germany. Tel: +497612039632; Fax: +497612039620; E-mail: ulrich.maurer@mol-med.uni-freiburg.de

Received 09.11.16; revised 03.2.17; accepted 13.2.17; Edited by M Gyrd-Hansen; published online 10.3.17 
The discovery of a second complex, induced by TNF, helped understanding an alternative activity of TNF, which is the induction of cell death by apoptosis. ${ }^{6}$ This complex, termed complex II, is derived from complex I and consists of RIPK1, FADD, caspase-8 and c-FLIP. Complex II provides the platform to activate caspase-8, which in turn results in caspase-3 processing and the induction of apoptosis. In general, proapoptotic caspase activation in complex II is repressed by TNF-induced transcription, that is, mediating the induction of c-FLIP. ${ }^{7}$ However, under certain circumstances, complex II (in this case termed complex $\mathrm{Ilb}$ ) can also induce apoptosis, which cannot be counteracted by c-FLIP, but requires the kinase activity of RIPK1.

RIPK1 is also required for TNF-induced programmed necrosis, termed necroptosis, representing an alternative form of TNF-induced cell death. ${ }^{9}$ The kinase activity of RIPK1 and its interaction in amyloid-like oligomers with RIPK3 mediates the phosphorylation and activation of the pseudokinase MLKL in order to execute necroptotic cell death. ${ }^{10}$ While apoptosis is executed by caspases, caspase activity prevents necroptosis. ${ }^{11}$ The requirement of caspase- 8 for the prevention of necroptosis was demonstrated on a genetic level, by the developmental rescue of mice lacking caspase-8, which die during mid-gestation, upon the additional absence of RIPK3 in casp8 ${ }^{-/-}$rip3 $^{-/-}$mice ${ }^{12-14}$ (Figure 1).

Regulated ubiquitylation and de-ubiquitylation is critical not only for the assembly and stability of the TNFR1 signalling complexes, but also for the decision between proinflammatory and prodeath signalling by TNF. The process of de-ubiquitylation is mediated by deubiquitinases (DUBs), which degrade or trim polyubiquitin chains. These enzymes, just as ubiquitin ligases, have specificities for certain target proteins and types of ubiquitin linkages.

The DUBs CYLD and OTULIN were shown to negatively regulate TNFR1-induced inflammatory signalling. Both DUBs were demonstrated to interact with the LUBAC complex and to counteract its activity, however their mechanism of action is different.

OTULIN specifically hydrolyses M1-liked ubiquitin chains, thereby directly counteracting the activity of LUBAC for the assembly of the TNF receptor complex. This results in the attenuation of TNF-induced NF- $\kappa \mathrm{B}$ activation. ${ }^{15,16}$ OTULIN directly interacts with LUBAC through the peptide:N-glycanase/UBA-or UBX-containing proteins (PUB) domain of HOIP, which in turn interacts with a PUB-interacting motif (PIM) in OTULIN. ${ }^{17,18}$ Thereby, OTULIN was shown to constitutively restrict M1-ubiquitylation of LUBAC components. ${ }^{19}$ Whether OTULIN exhibits DUB activity within the receptor complexes is a matter of debate. While M1-ubiquitylation of the TNF-RSC and the NOD2-RSC remained unchanged in cells lacking or retaining OTULIN in one study, ${ }^{19}$ another study observed increased TNFR1 - and RIPK2 ubiquitylation in OTULIN knockdown cells upon stimulation of the respective receptors. ${ }^{15}$

Like OTULIN, CYLD has important roles in inflammatory signalling. This enzyme specifically hydrolyses both K63- and M1-linked polyubiquitin chains. ${ }^{20,21}$ Importantly, upon stimulation of the respective receptors, CYLD is recruited to the TNF-RSC or the NOD2 signalling complex (NOD2-SC), limiting their ubiquitylation. CYLD is recruited to these receptor complexes along with LUBAC, by the interaction of CYLD with the LUBAC subunit HOIP. ${ }^{19}$ Just like the HOIP-OTULIN interaction, the HOIP-CYLD interaction was shown to require the PUB domain of HOIP. ${ }^{19,22,23}$ CYLD restricts TNFR1- or NOD2-induced ubiquitylation and NF- $k$ B activation, ${ }^{22,24-26}$ but it was also demonstrated to play an important role in the context of TNFR1-induced cell death. A siRNA screen identified CYLD to be crucial for both TNFR1-induced apoptosis and necroptosis, while another study had shown that CYLD was required for RIPK1-dependent apoptosis. ${ }^{8,27}$ Remarkably, CYLD is a substrate for cleavage by caspase-8 and the cleavage of CYLD was reported to underlie the anti-necroptotic activity of caspase- $8 .^{28}$

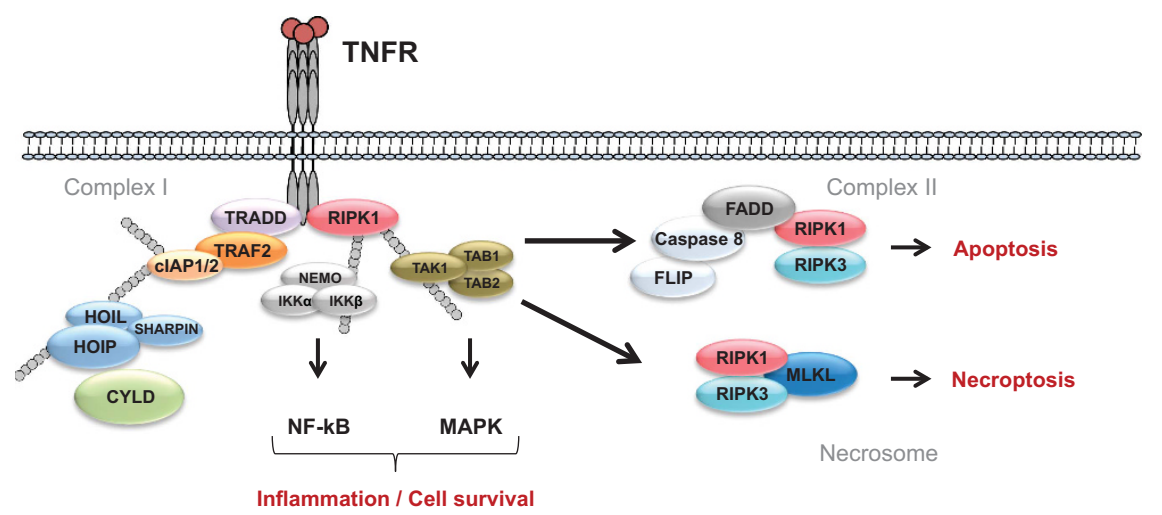

Figure 1 TNFR1 signalling. The binding of TNF to TNFR1 induces the formation of a protein complex called complex I. The assembly of this complex is initiated with the recruitment of TRADD and RIPK1 to the trimerised receptor. The proteins TRAF2 and CIAP1/2 are then recruited, generating K63-linked ubiquitin chains within the complex, which serve as a platform for the subsequent recruitment of TAB1/2 together with TAK1. In addition, the LUBAC complex (HOIL-1, HOIP, and SHARPIN) is recruited by these chains. By being associated with LUBAC, the DUB CYLD is also recruited to the complex. The E3 ligase activity of HOIP mediates linear ubiquitylation of different components within the complex, promoting complex I stabilisation and the recruitment of IKK $\alpha / \mathrm{IKK} \beta$ through its adaptor NEMO, which mediate NF $\kappa \mathrm{B}$ transcription factor activity. Together with MAP kinase signalling induced by TAK1, this results in proinflammatory, prosurvival gene expression. Under certain circumstances (e.g. loss of clAP1/2 or TAK1 activity) a second complex, termed complex II (consisting of the proteins RIPK1, RIPK3, FADD, caspase-8, and FLIP) can form, which is able to activate caspase-8 and induce apoptosis. TNFR1 stimulation upon inhibition of caspase-8 promotes the assembly of a complex consisting of RIPK1, RIPK3, and MLKL, which can lead to necroptotic cell death 
A novel molecule, involved in these signalling processes has now come into play. On the basis of different mass spectrometry (MS) approaches, four different studies have identified the protein SPATA2 as a novel component of the TNFR1 signalling complex. ${ }^{29-32}$

The initial report by Wagner et al. (2016) identified SPATA2, employing SILAC-MS, by immunoprecipitation of the TNF-RSC and analysis of the associated proteins. In a subsequent screen for proteins interacting with SPATA2 they identified CYLD, as well as the LUBAC components HOIL-1, HOIP, and SHARPIN, as SPATA2-binding proteins. ${ }^{29}$

Conversely, in our study, we performed a MS analysis to identify proteins interacting with CYLD, and SPATA2 stood out by exhibiting the highest enrichment with CYLD among all interacting proteins. ${ }^{30}$ Confirming the data of Wagner et al. (2016), a report by Kupka et al. ${ }^{31}$ showed that SPATA2 is a constituent of the TNF-RSC, and a binding partner of HOIP. A study by Eliott et al. (2016) identified SPATA2 by comparing the interactome of CYLD to the interactome of a CYLD mutant lacking the B-Box, which had been previously shown to be required for the interaction of CYLD with $\mathrm{HOIP}^{32}$

These findings are all consistent with an earlier observation, based on MS, of a CYLD-SPATA2 interaction. ${ }^{33}$ However, these recent studies now provide insight into the function of SPATA2. All four reports mapped the CYLD-SPATA2 interaction to the C-terminal ubiquitin-specific protease (USP) domain of CYLD and the N-terminus of SPATA2, which contains a PUB domain. Gyrd-Hansen and colleagues went a step further as they demonstrated, on a structural level, that this non-canonical PUB domain of SPATA2 binds to an interaction motif in CYLD, which is different from a typical PUB interaction motif (PIM). Importantly, their data further suggested that a dimerisation of the CYLD B-box results in a reduced $k_{\text {off }}$ for the interaction with SPATA2, promoting the formation of a CYLD-SPATA2 heterotetramer. Interestingly, this report identified the protein SPATA2L, a protein encoded by a separate gene, also as a B-box dependent binding partner of CYLD. However, SPATA2L could not compensate for SPATA2 functions, which are described below. ${ }^{32}$

CYLD had previously been shown to interact with HOIP, which mediates the recruitment of CYLD to the TNF-RSC upon TNFR1 stimulation. ${ }^{19}$ Consistent with the CYLDSPATA2 interaction, which suggests that SPATA2 associates with HOIP as well, a SPATA2-HOIP interaction was identified by mass spectrometry in two studies, ${ }^{29,31}$ while the two other studies found an association of SPATA2 and HOIP by Co-IP. ${ }^{30,32}$

Thus, it was well possible that SPATA2 interacts with HOIP directly. As described above, OTULIN directly interacts with the PUB domain of HOIP, which requires the PUB-interacting motif (PIM) of OTULIN. ${ }^{17,18}$ This raised the possibility that SPATA2, just as OTULIN, binds to HOIP through a PIM-PUB interaction. Indeed, we found that SPATA2 contains a PIM, and the mutation of a critical amino acid (Y338) within the PIM resulted in the loss of the interaction of SPATA2 with HOIP (but not CYLD). The same was observed by Elliott et al. (2016) and their structural analysis again provided a deeper insight into this interaction, demonstrating a high homology of the respective PIM in OTULIN and SPATA2, with a high specificity for the PUB domain of HOIP (Figure 2).

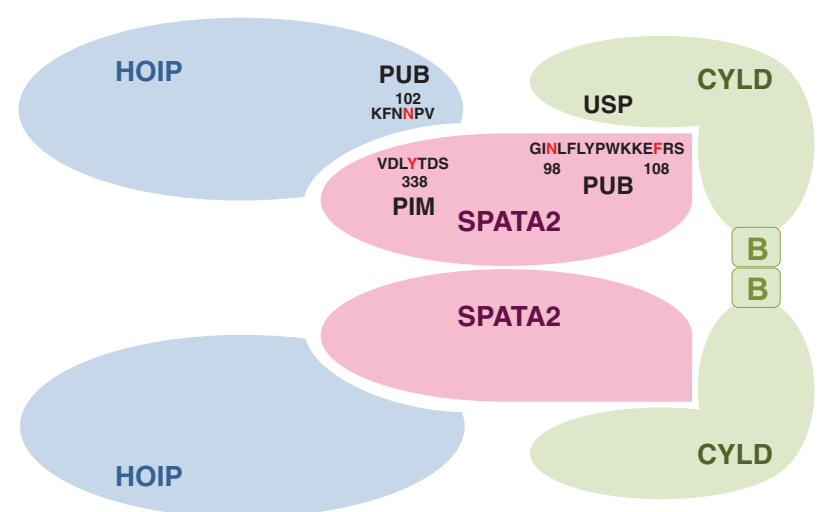

Figure 2 Interaction of SPATA2 with CYLD and HOIP. SPATA2 represents the link between the proteins CYLD and HOIP, binding to each protein through a distinct domain. The PUB domain of SPATA2, with the crucial amino acids N98 and F108, binds the USP domain of CYLD. CYLD itself forms a homodimer via a B-box/B-box interaction, which in consequence leads to a heterotetramer composed of two CYLD and two SPATA2 proteins. SPATA2 contains a PIM (with the crucial amino acid Y338) that directly interacts with the PUB domain of HOIP (an essential amino acid being N102). These interactions promote the assembly of a complex consisting of a CYLDSPATA2 heterotetramer interacting with two LUBAC (HOIL-1, HOIP, and SHARPIN) complexes

Thus, as SPATA was found to bind to both CYLD (via its PUB domain) and HOIP (through its PIM), it was a candidate to mediate the interaction of HOIP and CYLD. Indeed, the interaction of CYLD and HOIP was shown to be indirect in the studies by Kupka et al. (2016) and Elliott et al. (2016)..$^{31,32}$ Consistent with the idea that SPATA2 is the bridging factor between CYLD and HOIP, the recruitment of CYLD to the TNF-RSC was compromised in cells lacking SPATA2. ${ }^{29-32}$ Moreover, SPATA2 was not recruited to the TNF-RSC in HOIP-deficient cells, further demonstrating that SPATA2 provides the link for the recruitment of CYLD to the TNF-RSC, by LUBAC (Figure 3). This mechanism extends beyond TNFR1 signalling, as SPATA2 was also found to be recruited to the NOD2 signalling complex in a HOIP-dependent manner. ${ }^{31}$

CYLD and OTULIN were demonstrated to be bound to HOIP in mutually exclusive manner. ${ }^{19}$ This can be explained now by the findings showing that the PIM of SPATA2, just like the PIM of OTULIN, binds the PUB domain of HOIP. Indeed, our experiments showed that SPATA2 and OTULIN compete for binding to HOIP. ${ }^{30}$

Moreover, CYLD, but not OTULIN, was reported to be recruited to the TNF-RSC and NOD2-SC by LUBAC. ${ }^{19}$ Thereby CYLD would exhibit its DUB activity within the receptor complexes, while OTULIN counteracts receptor activation-independent basal ubiquitylation activity of free cytosolic LUBAC. Possibly, the interaction with OTULIN prevents the recruitment of LUBAC to receptor complexes, while the interaction of LUBAC with SPATA2 (and CYLD) does not. It should be noted, however, that Wagner et al. (2016) found a slight enrichment of OTULIN with the TNF-RSC, ${ }^{29}$ confirming previous similar data by others. ${ }^{18}$ Moreover, an association of OTULIN with the NOD2-SC has been reported, ${ }^{15,22}$ which would argue against an exclusive recruitment of the CYLD-SPATA2-LUBAC complex to receptors. 
a

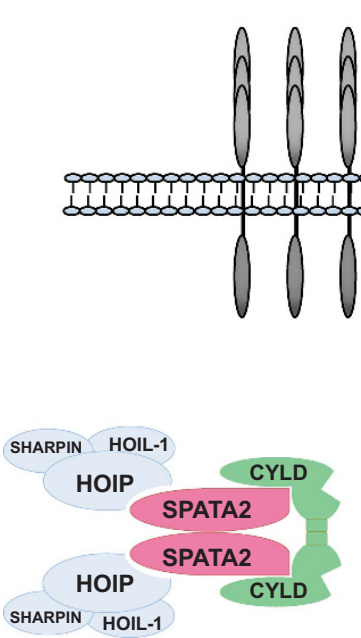

b

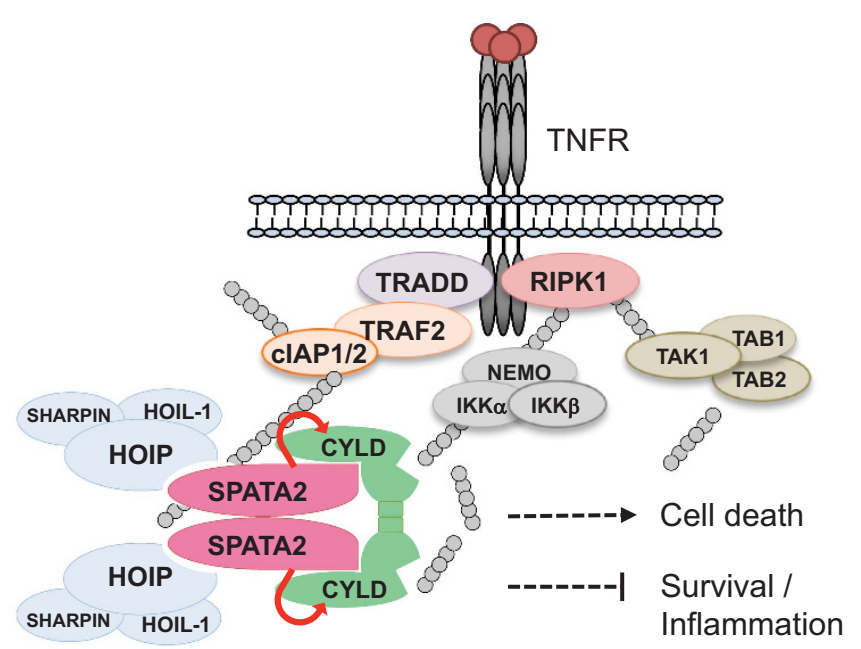

Figure 3 Role of SPATA2 within the TNF-RSC. (a) The CYLD/SPATA2/LUBAC complex is pre- existing in the cytosol, in absence of TNF stimulation. (b) Upon TNF stimulation and complex I assembly, the CYLD/SPATA2/LUBAC complex is recruited to the receptor complex, via interaction of LUBAC to K63-linked ubiquitin chains present in the receptor complex. Here SPATA2 promotes CYLD DUB activity, thereby mediating the de-ubiquitylation of different complex components such as RIPK1. Activated CYLD thereby limits proinflammatory signalling and favours complex II formation, leading to TNF-induced cell death

However, SPATA2 is more than an adaptor for the recruitment of CYLD to the TNF-RSC. Intriguingly, the direct interaction of SPATA2 with the USP domain of CYLD entailed an increase of the enzymatic activity of CYLD, as indicated by the enhanced cleavage of M1- and K63-linked di- or tetra-ubiquitin substrates. $^{30,32}$ Thus, SPATA2 is an allosteric activator of CYLD. This mode of regulation is not unique to CYLD, as an allosteric activation was recently shown for the DUB USP 12. ${ }^{34}$

Taken together, SPATA2 is crucial for the DUB activity of CYLD in the TNF-RSC and NOD2-SC, by CYLD recruitment and activation. Therefore, loss of SPATA2 should translate into increased ubiquitylation at the TNF-RSC, and consistently, we observed increased M1-linked ubiquitylation of the TNF-RSC in SPATA2 ko cells. Unexpectedly, Wagner et al. (2016) found less ubiquitylation of RIPK1 in absence of SPATA2. Similarly, Eliott et al. (2016) observed reduced amounts of ubiquitylated RIPK1, TNFR1, as well as total K63-ubiquitin and M1-ubiquitin chains in the TNF-RSC in cells lacking SPATA2. In contrast, in TNF-stimulated cells lacking CYLD, an increase of M1ubiquitylated RIPK1, TNFR1 and TRADD in the TNF-RSC had been observed, ${ }^{19}$ raising the possibility that SPATA2 has also CYLD-independent effects. However, upon NOD2 stimulation, SPATA2 ko cells, similar to CYLD ko cells, exhibited increased ubiquitylation of RIPK1 and RIPK2. ${ }^{32}$

Similar to the effects on ubiquitylation, the effects of SPATA2 on TNF-induced NF-KB and MAPK signalling exhibited quite some variation depending on the stimulus and the respective study. Increased NF- $k$ B activation in cells lacking SPATA2 was observed by Wagner et al. (2016) and supported by our data as well, ${ }^{30}$ while the absence of SPATA2 had no effect on TNFinduced NF- $K$ B activation in the two other studies. ${ }^{31,32}$ A slight activation of MAPK upon TNF stimulation was seen by Kupka et al (2016), which appeared more pronounced in our experiments, ${ }^{30}$ while the elevated MAPK induction was not observed in the two remaining reports. ${ }^{29,32}$ Why those different effects, caused by the absence of SPATA2, on proinflammatory TNF signalling were observed is not clear, but this seems somewhat reminiscent of the heterogeneous effects on NF- $K B$ activation by TNF, which were observed in absence of CYLD with different studies. ${ }^{19,24,26,35,36}$

However, the attenuating role of CYLD in the context of NOD2 proinflammatory signalling, as shown previously, ${ }^{22}$ is clearly reflected by SPATA2, as its absence promoted ubiquitylation of $\mathrm{RIPK} 2$, resulting in elevated $\mathrm{NF}-K \mathrm{~B}$ activation and induction of inflammatory cytokines upon NOD2 stimulation with L18-MDP. ${ }^{32}$

The perhaps most intriguing effect observed with cells lacking SPATA2 was the profound reduction of TNF-induced cell death. We found that the additional absence of SPATA2 in $\mathrm{TAK}^{-1}{ }^{-}$cells, which are permissive for TNF-induced, RIPK1dependent apoptosis, largely reduced the formation of TNFR1 complex II upon treatment with TNF. Accordingly, caspase-3 processing and apoptosis was diminished in these cells in absence of SPATA2, indicating an important role of SPATA2 for TNF-induced apoptosis. ${ }^{30}$

Moreover, Wagner et al. (2016) showed a reduction of TNF/ZVAD-induced necroptosis in L929 cells when SPATA2 was knocked down, which was confirmed by the data of Kupka et al. (2016). The former study also showed that the reduction of necroptosis in SPATA2 knockdown cells was associated with a loss of MLKL phosphorylation under these conditions.

Notably, the observation that the absence of SPATA2 prevented TNF-induced cell death had been made already earlier. ${ }^{27}$ While not further analysed in this study, SPATA2, similar to CYLD, was identified by a siRNA screen as required for both TNF-induced apoptosis and necroptosis of L929 cells.

Taken together, these data show an important role of SPATA2 for TNF-induced cell death and suggest a functional similarity between CYLD and SPATA2 in this context, not only for signalling through TNFR complex I, but also for the assembly of TNFR complex II (Figure 3).

However, things are possibly more complicated, as the death-promoting function of SPATA2 might be not exclusively 
connected to CYLD: previous work by others showed that apoptosis induced by TNF upon inhibition of TAK1 with (5Z)-7Oxozeaenol could not be prevented by knockdown of CYLD. ${ }^{37}$ Consistent with these data, we observed that CRISPR/Cas9mediated loss of CYLD did not prevent the death of TNF-treated TAK $1^{-/-}$cells (unpublished data). However, the absence of SPATA2 was rate-limiting for TNF-induced apoptosis of $\mathrm{TAK}^{-1-}$ cells. $^{30}$ Thus, not all functions of SPATA2 for the promotion of apoptosis can be explained by its recruitment of CYLD to receptor complexes, that is, SPATA2 apparently has functions in TNF-induced cell death that go beyond promoting CYLD activity.

SPATA2 (back then named PD1) cDNA was first isolated from a testis library and shown to be highly expressed in testicular Sertoli cells. ${ }^{38}$ Testis are considered an immuneprivileged site, but Sertoli cells are capable of initiating an innate immune response through TLR signalling. ${ }^{39}$ It is possible that SPATA2 attenuates TLR signalling and inflammatory cytokine expression in this tissue by promoting CYLD activity. Interestingly, $\mathrm{CYLD}^{-/-}$male mice were described to be infertile due the absence of an early wave of germ cell apoptosis, required for subsequent productive spermatogenesis, ${ }^{40}$ and it an interesting question whether SPATA2 is required for male fertility in mice.

It will be important to address whether mice deficient for SPATA2 would exhibit a similar phenotype as observed with $\mathrm{CYLD}^{-/-}$animals, such as T-cell hyperactivation or elevated responsiveness of B-cells and macrophages. ${ }^{36,41}$ Moreover, given the crucial role of SPATA2 for TNF-induced cell death, it is tempting to speculate that the absence of SPATA2 could rescue the mid-gestation developmental defects of mice deficient for TAK1 or caspase-8, which have been shown to depend on TNFR1 signalling. ${ }^{42,43}$

Furthermore, as SPATA2 may have CYLD-independent functions, it remains to be clarified whether SPATA2 interacts with other proteins in the TNF receptor complex under conditions resulting in cell death. Together, the growing number of proteins being instrumental for inflammatory signalling pathways is now extended by SPATA2, and further characterisation of the role of SPATA2 will certainly help to understand the complexity of immune receptor signalling.

\section{Conflict of Interest}

The authors declare no conflict of interest.

Acknowledgements. Work in our lab is supported by the Centre for Biologica Signalling Studies (BIOSS), Hebelstrasse 2, D-79104, Freiburg, Germany, funded by the Excellence Initiative, Germany (EXC-294), and the Spemann Graduate School of Biology and Medicine (SGBM), Albert-Ludwigs-University of Freiburg, Albertstrasse 19a, D-79104, Freiburg, Germany, funded by the Excellence Initiative of the German Federal and State Governments, Germany (GSC-4), and grant 112140 from the Deutsche Krebshilfe to UM. We apologise to the colleagues whose contributions have not been cited due to space constraints.

1. Takeuchi O, Akira S. Pattern recognition receptors and inflammation. Cell 2010; 140: 805-820.

2. Emmerich $\mathrm{CH}$, Ordureau A, Strickson S, Arthur JSC, Pedrioli PGA, Komander D et al. Activation of the canonical IKK complex by K63/M1-linked hybrid ubiquitin chains. Proc Natl Acad Sci USA 2013; 110: 15247-15252.

3. Ohtake F, Saeki Y, Ishido S, Kanno J, Tanaka K. The K48-K63 Branched Ubiquitin Chain Regulates NF-kB Signaling. Mol Cell 2016; 64: 251-266.
4. Wang C, Deng L, Hong M, Akkaraju GR, Inoue J, Chen ZJ. TAK1 is a ubiquitin-dependent kinase of MKK and IKK. Nature 2001; 412: 346-351.

5. Rahighi S, Ikeda F, Kawasaki M, Akutsu M, Suzuki N, Kato R et al. Specific recognition of linear ubiquitin chains by NEMO is important for NF-kappaB activation. Cell 2009; 136: 1098-1109.

6. Micheau O, Tschopp J. Induction of TNF receptor I-mediated apoptosis via two sequential signaling complexes. Cell 2003; 114: 181-190.

7. Micheau O, Lens S, Gaide O, Alevizopoulos K, Tschopp J. NF-kappaB signals induce the expression of c-FLIP. Mol Cell Biol 2001; 21: 5299-5305.

8. Wang L, Du F, Wang X. TNF-alpha induces two distinct caspase-8 activation pathways. Cell 2008; 133: 693-703.

9. Holler N, Zaru R, Micheau O, Thome M, Attinger A, Valitutti S et al. Fas triggers an alternative, caspase-8-independent cell death pathway using the kinase RIP as effector molecule. Nat Immunol 2000; 1: 489-495.

10. Sun L, Wang H, Wang Z, He S, Chen S, Liao D et al. Mixed lineage kinase domain-like protein mediates necrosis signaling downstream of RIP3 kinase. Cell 2012; 148: 213-227.

11. Vercammen D, Beyaert R, Denecker G, Goossens V, Van Loo G, Declercq W et al. Inhibition of caspases increases the sensitivity of L929 cells to necrosis mediated by tumor necrosis factor. J Exp Med 1998; 187: 1477-1485.

12. Varfolomeev EE, Schuchmann M, Luria V, Chiannilkulchai N, Beckmann JS, Mett IL et al. Targeted disruption of the mouse Caspase 8 gene ablates cell death induction by the TNF receptors, Fas/Apo1, and DR3 and is lethal prenatally. Immunity 1998; 9: 267-276.

13. Oberst A, Dillon CP, Weinlich R, McCormick LL, Fitzgerald P, Pop C et al. Catalytic activity of the caspase-8-FLIP(L) complex inhibits RIPK3-dependent necrosis. Nature 2011; 471: 363-367.

14. Kaiser WJ, Upton JW, Long AB, Livingston-Rosanoff D, Daley-Bauer LP, Hakem R et al. RIP3 mediates the embryonic lethality of caspase-8-deficient mice. Nature 2011; 471: 368-372.

15. Fiil BK, Damgaard RB, Wagner SA, Keusekotten K, Fritsch M, Bekker-Jensen S et al. OTULIN restricts Met1-linked ubiquitination to control innate immune signaling. Mol Cell 2013; 50: 818-830.

16. Keusekotten K, Elliott PR, Glockner L, Fill BK, Damgaard RB, Kulathu Y et al. OTULIN antagonizes LUBAC signaling by specifically hydrolyzing Met1-linked polyubiquitin. Cell 2013; 153: 1312-1326.

17. Elliott PR, Nielsen SV, Marco-Casanova P, Fiil BK, Keusekotten K, Mailand N et al. Molecular basis and regulation of OTULIN-LUBAC interaction. Mol Cell 2014; 54: 335-348.

18. Schaeffer V, Akutsu M, Olma MH, Gomes LC, Kawasaki M, Dikic I. Binding of OTULIN to the PUB Domain of HOIP Controls NF-kappa B Signaling. Mol Cell 2014; 54: 349-361.

19. Draber P, Kupka S, Reichert M, Draberova H, Lafont E, de Miguel D et al. LUBAC-recruited CYLD and $A 20$ regulate gene activation and cell death by exerting opposing effects on linear ubiquitin in signaling complexes. Cell Rep 2015; 13: 2258-2272.

20. Komander D, Reyes-Turcu F, Licchesi JDF, Odenwaelder P, Wilkinson KD, Barford D. Molecular discrimination of structurally equivalent Lys 63-linked and linear polyubiquitin chains. EMBO Rep 2009; 10: 466-473.

21. Ritorto MS, Ewan R, Perez-Oliva AB, Knebel A, Buhrlage SJ, Wightman M et al. Screening of DUB activity and specificity by MALDI-TOF mass spectrometry. Nat Commun 2014; 5: 4763.

22. Hrdinka M, Fiil BK, Zucca M, Leske D, Bagola K, Yabal M et al. CYLD limits Lys63- and Met1linked ubiquitin at receptor complexes to regulate innate immune signaling. Cell Rep 2016; 14: $2846-2858$.

23. Takiuchi T, Nakagawa T, Tamiya H, Fujita H, Sasaki Y, Saeki Y et al. Suppression of LUBACmediated linear ubiquitination by a specific interaction between LUBAC and the deubiquitinases CYLD and OTULIN. Genes Cells 2014; 19: 254-272.

24. Kovalenko A, Chable-Bessia C, Cantarella G, Israël A, Wallach D, Courtois G. The tumour suppressor CYLD negatively regulates NF-kappaB signalling by deubiquitination. Nature 2003; 424: 801-805

25. Trompouki E, Hatzivassiliou E, Tsichritzis T, Farmer H, Ashworth A, Mosialos G. CYLD is a deubiquitinating enzyme that negatively regulates NF-kappaB activation by TNFR family members. Nature 2003; 424: 793-796.

26. Brummelkamp TR, Nijman SMB, Dirac AMG, Bernards R. Loss of the cylindromatosis tumour suppressor inhibits apoptosis by activating NF-kappaB. Nature 2003; 424: 797-801.

27. Hitomi J, Christofferson DE, Ng A, Yao J, Degterev A, Xavier RJ et al. Identification of a molecular signaling network that regulates a cellular necrotic cell death pathway. Cell 2008; 135: 1311-1323.

28. O'Donnell MA, Perez-Jimenez E, Oberst A, $\mathrm{Ng} \mathrm{A}$, Massoumi $\mathrm{R}$, Xavier $\mathrm{R}$ et al. Caspase 8 inhibits programmed necrosis by processing CYLD. Nat Cell Biol 2011; 13: 1437-1442.

29. Wagner SA, Satpathy S, Beli P, Choudhary C. SPATA2 links CYLD to the TNF- $\alpha$ receptor signaling complex and modulates the receptor signaling outcomes. EMBO $J$ 2016; 35 : 1868-1884.

30. Schlicher $L$, Wissler $M$, Preiss $F$, Brauns-Schubert $P$, Jakob $C$, Dumit $V$ et al. SPATA2 promotes CYLD activity and regulates TNF-induced NF- $\mathrm{KB}$ signaling and cell death. EMBO Rep 2016; 17: 1485-1497.

31. Kupka S, de Miguel D, Draber P, Martino L, Surinova S, Rittinger $K$ et al. SPATA2-mediated binding of CYLD to HOIP enables CYLD recruitment to signaling complexes. Cell Rep 2016; 16: $2271-2280$.

32. Elliott PR, Leske D, Hrdinka M, Bagola K, Fiil BK, McLaughlin SH et al. SPATA2 Links CYLD to LUBAC, activates CYLD, and controls LUBAC signaling. Mol Cell 2016; 63: 990-1005. 
33. Sowa ME, Bennett EJ, Gygi SP, Harper JW. Defining the human deubiquitinating enzyme interaction landscape. Cell 2009; 138: 389-403.

34. Li H, Lim KS, Kim H, Hinds TR, Jo U, Mao H et al. Allosteric activation of ubiquitin-specific proteases by $\beta$-propeller proteins UAF1 and WDR20. Mol Cell 2016; 63: 249-260.

35. Lee CC, Carette JE, Brummelkamp TR, Ploegh HL. A reporter screen in a human haploid cell line identifies CYLD as a constitutive inhibitor of NF-кB. PLOS ONE 2013; 8: e70339.

36. Zhang J, Stirling B, Temmerman ST, Ma CA, Fuss IJ, Derry JMJ et al. Impaired regulation of NF-kappaB and increased susceptibility to colitis-associated tumorigenesis in CYLD-deficient mice. J Clin Invest 2006; 116: 3042-3049.

37. Dondelinger Y, Aguileta MA, Goossens V, Dubuisson C, Grootjans S, Dejardin E et al. RIPK3 contributes to TNFR1-mediated RIPK1 kinase-dependent apoptosis in conditions of cIAP1/2 depletion or TAK1 kinase inhibition. Cell Death Differ 2013; 20: 1381-1392.

38. Graziotto R, Foresta C, Scannapieco P, Zeilante P, Russo A, Negro A et al. cDNA cloning and characterization of PD1: a novel human testicular protein with different expressions in various testiculopathies. Exp Cell Res 1999; 248: 620-626.
39. Riccioli A, Starace D, Galli R, Fuso A, Scarpa S, Palombi F et al. Sertoli cells initiate testicular innate immune responses through TLR activation. J Immunol 2006; 177 7122-7130.

40. Wright A, Reiley WW, Chang M, Jin W, Lee AJ, Zhang M et al. Regulation of early wave of germ cell apoptosis and spermatogenesis by deubiquitinating enzyme CYLD. Dev Cell 2007; 13: $705-716$.

41. Reiley WW, Jin W, Lee AJ, Wright A, Wu X, Tewalt EF et al. Deubiquitinating enzyme CYLD negatively regulates the ubiquitin-dependent kinase Tak1 and prevents abnormal $T$ cell responses. J Exp Med 2007; 204: 1475-1485.

42. Morioka S, Inagaki M, Komatsu Y, Mishina Y, Matsumoto K, Ninomiya-Tsuji J. TAK1 kinase signaling regulates embryonic angiogenesis by modulating endothelial cell survival and migration. Blood 2012; 120: 3846-3857.

43. Dillon CP, Weinlich R, Rodriguez DA, Cripps JG, Quarato G, Gurung P et al. RIPK1 blocks early postnatal lethality mediated by caspase-8 and RIPK3. Cell 2014; 157 1189-1202. 